\title{
Influence of an Electric Field on Fine Properties of III-V and II-VI Quantum Dots Systems
}

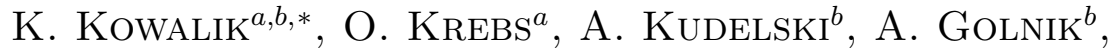 \\ A. Lemaître ${ }^{a}, \mathrm{P} . \mathrm{Senellart}^{a}, \mathrm{G} \cdot \mathrm{Karczewski}^{c}, \mathrm{~J} \mathrm{Kossut}^{c}$, \\ J. GAJ ${ }^{b}$ AND P. VOISIN ${ }^{a}$ \\ ${ }^{a}$ Laboratoire de Photonique et Nanostructures-CNRS \\ Route de Nozay, 91460 Marcoussis, France \\ ${ }^{b}$ Institute of Experimental Physics, Warsaw University \\ Hoża 69, 00-681 Warszawa, Poland \\ ${ }^{c}$ Institute of Physics, Polish Academy of Sciences \\ al. Lotników 32/46, 02-668 Warszawa, Poland
}

\begin{abstract}
We investigate the influence of an electric field on the optical properties of single quantum dots. For sample made of III-V compounds micron-size electro-optical structures were produced in order to apply an electric field in the dot plane. For several individual dots lines significant variations of the anisotropic exchange splitting with the field were observed. On sample made of II-VI compounds we demonstrate the influence of electric field fluctuations on the luminescence of a single quantum dot.
\end{abstract}

PACS numbers: 78.67.Hc, 71.70.Ej

\section{Introduction}

Semiconductor quantum dots (QDs) currently attract much attention in the field of photon-based quantum cryptography, because of their possible use as emitters of entangled photon pairs. Although polarization correlation has already been reported in the biexciton-exciton cascade of a single QD no real quantum entanglement has been achieved so far. One of the reason for that is the anisotropic exchange splitting (AES) of the intermediate state (the bright exciton) which turns

*corresponding author; e-mail: kkowalik@fuw.edu.pl 
out to be in general larger than the homogeneous line width of optical transitions, allowing two distinguishable paths for the biexciton recombination. The AES of excitons into linearly-polarized states is produced by some in-plane anisotropy of the individual self-assembled QDs, likely due to an elongation developing in a privileged direction during the growth process. Therefore, controlling the QD anisotropy by an external perturbation might be a prerequisite for the achievement of photon entanglement with QDs.

In this work we explored perspectives to compensate the AES by applying an electric field in the plane of a QD structure. This method, which is supported by theoretical estimations [1], is yet far from being intuitive. To reduce AES we need to compensate somehow the QD shape anisotropy which can be considered as the result of a perturbation having a $D$-type symmetry. For example, it couples the single-particle $P$-states with $\left|\Delta J_{z}\right|=2$. An in-plane electric field produces a $P$-type perturbation which couples states with $\left|\Delta J_{z}\right|=1$ and thus does not seem sufficient in order to compensate the anisotropy. But since it affects both the electron and hole wave functions, it may act as a $D$-type perturbation on the excitonic ground state. Using the formalism developed to calculate the long-range (LR) part of the electron-hole exchange [2,3], it can be shown that a significant reduction of AES can be obtained by applying an electric field parallel to the long axis of the elongated QD [1]. Obviously, the subsequent reduction of exciton oscillator strength by the Stark effect limits the amplitude of the field that can be used on that purpose. In practice it also demands a fine control of the electric field (in amplitude and direction), which may depend on the excitation conditions or on particular details of the sample. We addressed this issue by studying the influence of electric field on single QD photoluminescence (PL), on two different samples (A: InAs/GaAs QDs, and B: ZnTe/CdTe QDs) and for different external parameters.

\section{Investigation of InAs/GaAs QDs in a planar field-effect structure}

The III-V sample (A) was grown by molecular beam epitaxy in the Laboratory for Photonics and Nanostructures. It contains a single plane of self-assembled InAs quantum dots in the center of a $200 \mathrm{~nm}$ thick GaAs layer, sandwiched between two $20 \mathrm{~nm}$ thick barriers of $\mathrm{Ga}_{0.8} \mathrm{Al}_{0.2} \mathrm{As}$ to prevent carrier diffusion. In order to apply an electric field in the plane of the QDs, two metallic contacts were deposited on the surface: (i) an $n$-type ohmic contact obtained by annealing a $\mathrm{Ni} / \mathrm{Ge} / \mathrm{Au}$ multilayer, which acts as a deep reference electrode, (ii) a $\mathrm{Ti} / \mathrm{Au}$ Schottky contact. Both electrodes were separated by a $2 \mu \mathrm{m}$ wide channel providing the desired in-plane field geometry and working also as an optical mask helpful for single QD spectroscopy. In order to make the required electrical bondings, the sample was glued on a ceramic holder which in turn was attached to the 
cold finger of a liquid helium flux cryostat assuring a temperature below $20 \mathrm{~K}$. For PL excitation, a tunable cw Ti:sapphire laser was used together with a micro-PL setup based on a $\times 50$ microscope-objective of long working distance. The typical value of AES in InAs QDs are in the $50 \mu \mathrm{eV}$ range. Its straightforward measurement demands thus a spectral resolution difficult to obtain with standard PL spectroscopy [4]. This difficulty can be overcome by using polarization-resolved spectroscopy. Indeed both components of the excitonic doublet split by the exchange interaction are linearly polarized along orthogonal directions, usually along the crystallographic axes $\langle 110\rangle$ for InAs/GaAs QDs. Our method of measurement relies thus on the PL line shift of a single QD when rotating a linear analyzer. In practice we rotated a half-wave plate placed in front of a fixed Glan-Thomson polarizer. We checked that this procedure by itself does not introduce any line shift. The PL signal was filtered by a double monochromator and detected with a liquid-nitrogen-cooled CCD array, providing a spectral resolution of $40 \mu \mathrm{eV}$. Using a line shape analysis procedure, we were able to determine the position of narrow lines with a precision better than $10 \mu \mathrm{eV}$ in the spectral region of interest.

Typical micro-PL spectra of a set of a few QDs excited at $1.36 \mathrm{eV}$ are shown in Fig. 1 for different applied bias $V_{\mathrm{g}}$. The Stark effect which manifests itself by the energy shift of the exciton ground state with the bias is the straightforward indication that an electric field is applied in the QD region with the employed technique. The PL spectra exhibit some lines that show a well defined Stark shift and some others that do not or start to shift at higher bias. This indicates that the electric field is not homogeneous along the small region $\left(\approx 2 \mu \mathrm{m}^{2}\right)$ excited by the laser spot. Also, the effective field experienced by the QD does not correspond to the nominal value $F_{\mathrm{n}}=\left(V_{\mathrm{g}}+V_{\mathrm{S}}\right) / L$, where $L$ is the distance between both electrodes and $V_{\mathrm{S}}$ the height of the Schottky barrier. Indeed, the observed Stark shift remains rather small (of the order of $100 \mu \mathrm{eV}$ at $F_{\mathrm{n}}=30 \mathrm{kV} / \mathrm{cm}$ ), compared with usually observed values in vertical field-effect structures [5] $(\approx 300 \mu \mathrm{eV}$ at $50 \mathrm{kV} / \mathrm{cm}$ ), although it should be more pronounced in the in-plane geometry be-

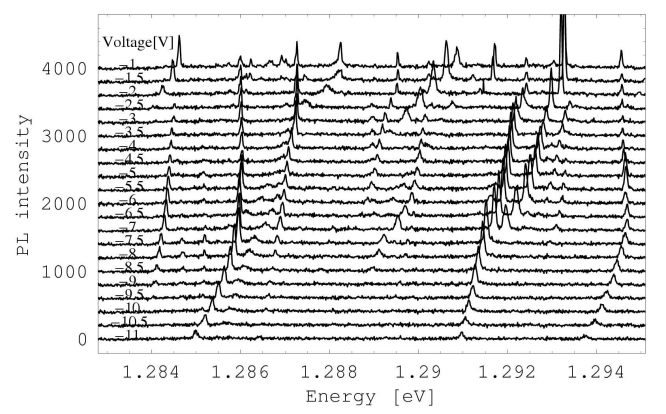

Fig. 1. Micro-PL spectra of a few InAs QDs at different applied voltages. Spectra are shifted for clarity. 
cause of the larger lateral dimensions of the dots. A few meV would be expected from a first-order estimate made in the effective mass approximation. We conclude that the QD plane tends to remain nearly equipotential and most of the potential drop occurs in the region below the Schottky electrode. This is a difficulty associated with the chosen technology: it is likely that a planar $p-i-n$ structure would allow an easier control of the in-plane electric field, at the expense of more stringent control of ohmic contact annealing.

Nevertheless, varying the optical excitation energy from $1.36 \mathrm{eV}$ to $1.42 \mathrm{eV}$, i.e. from an intra-dot (or quasi-resonant) to a non-resonant excitation regime in the $2 \mathrm{D}$ continuum of the $\mathrm{QD}$ wetting layer (WL), supports the idea that the in-
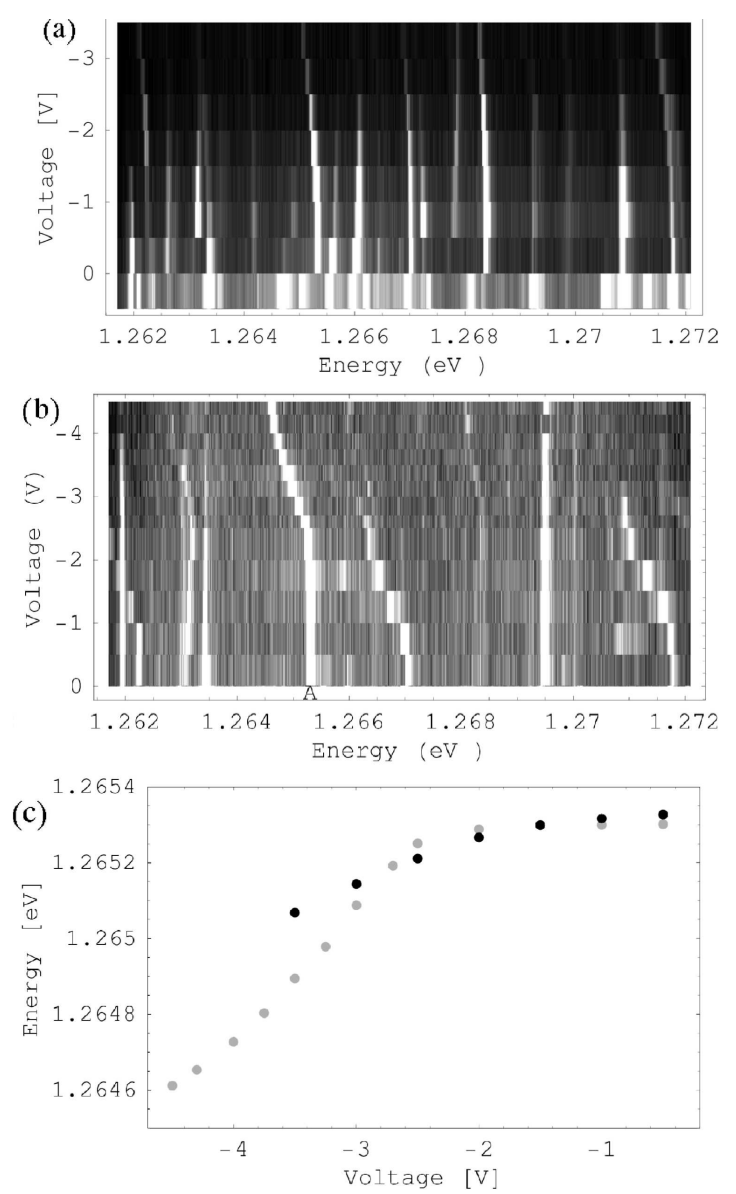

Fig. 2. Contour-plots in a gray scale of the PL intensity of InAs QDs as a function of the energy detection and applied bias: (a) under non-resonant excitation, (b) quasi-resonant excitation. (c) Energy position of the line labelled A against the bias under quasi-resonant (dark points) and non-resonant (gray points) excitation. 
-plane component of the electric field is not completely zero. Indeed, photocarriers created in the WL are swept very efficiently by such an in-plane electric field, leading to a dramatic decrease in their capture and thus of the PL intensity. This behavior is clearly observed in Figs. $2 \mathrm{a}$ and $\mathrm{b}$ that show on a gray scale the contour-plots of PL intensity against bias and energy: the PL intensity of lines decreases more rapidly with the bias under non-resonant excitation and the associated Stark shift (shown for line A in Fig. 2c) is smaller likely because of a stronger field-screening produced by the accumulation of photocarriers on both electrodes. As a consequence, we studied the field influence on AES mainly in the regime of intra-dot excitation which extends significantly the domain of useful field. Another advantage of this excitation regime is that it naturally selects a smaller number of QDs making the analysis of single lines easier.

As explained above, the AES is measured by polarization-resolved micro-PL spectroscopy, as the difference of energy positions between both components of the bright exciton doublet, supposed to be linearly polarized parallel to the cleaved edges of the sample. This property could be verified by rotating continuously the analyzer for some single QDs and was anyway quite obvious for many QDs with AES larger than the spectral resolution. Figure 3a shows polarization-resolved spectra with their Gaussian fit used to determine precisely the line positions. As
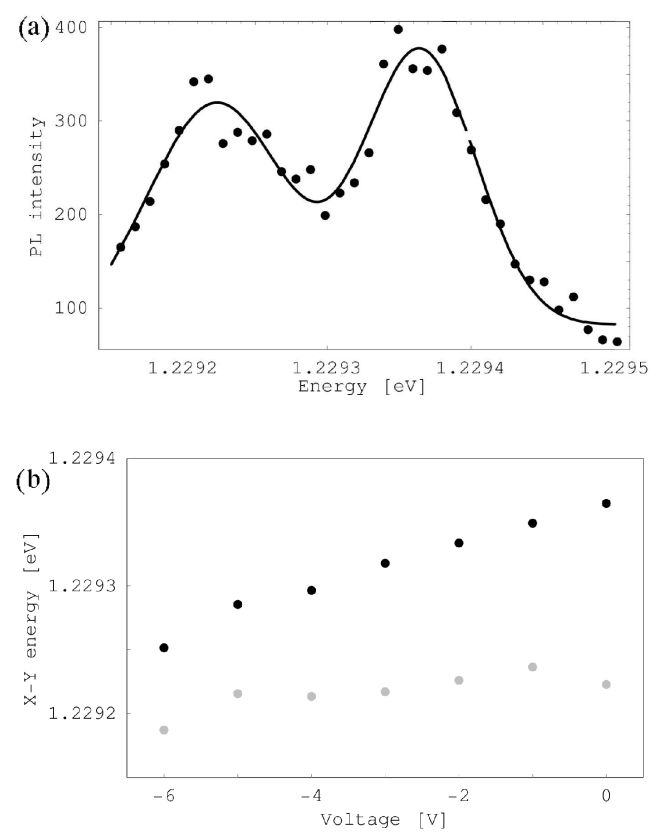

Fig. 3. (a) Examples of the $X$ - and $Y$-linearly polarized doublet of an exciton confined in a single anisotropic QD (dots), and lines analysis with a Gaussian fit (solid line). (b) Energy positions of the exciton $X$ - and $Y$-components (black and gray points) against the applied electric field bias. The $X-Y$ splitting (AES) decreases with the field. 
shown in Fig. 3b for a particular QD, the AES can be changed significantly with the applied bias. Here the reduction amounts to $\approx 77 \mu \mathrm{eV}$, i.e. half the initial value. The observed dependence is basically linear in the bias, although from simple symmetry considerations this effect should be even in the field. Since the observed Stark shift is also mainly linear, this result is not surprising. It again reveals the nonlinear relationship between the applied bias and the effective electric field. Yet, a similar behavior could also be produced by an asymmetric QD along the direction of the field. This AES reduction, though not systematically observed, could be observed on many different single QDs, but could not be followed up to the complete cancelling out of AES before the PL extinction. We can also mention that such a behavior was not observed with InAs QDs in a vertical field-effect structure that we studied during the same period. The current results obtained with a structure which can be obviously improved, support the perspective of using an in-plane electric field to control and possibly cancel out the AES.

\section{Observation of optically-induced Stark shift in $\mathrm{CdTe} / \mathrm{ZnTe}$ single QDs}

In this section, we present preliminary results of a study carried out on $\mathrm{CdTe} / \mathrm{ZnTe}$ single QDs. This sample was grown in the Institute of Physics, Polish Academy of Sciences in Warsaw [6]. Its structure consists in a single layer of CdTe QDs deposited by atomic layer epitaxy (ALE) between two ZnTe barriers. Its detailed description and basic characterization can be found in [7]. Microluminescence measurements were carried out in a magneto-optical cryostat at superfluid helium temperature. High numerical aperture and mechanical stability of the micro-PL setup were assured by a specially designed objective and sample holder [8]. The $488 \mathrm{~nm}$ emission line of a multi-line $\mathrm{UV} \mathrm{Ar}^{+}$laser was used for excitation. A more precise description of the experimental setup is given in [9].

Although no controlled external field could be applied in this structure, we report micro-PL characterization indicating the possibility of modifying the local field experienced by a QD by changing the excitation conditions. Figure 4 shows the temporal evolution of PL spectra for different power densities. The detection energy was set in the lower part of the QD PL distribution in order to spectrally select single QDs. When the excitation power increases, the lines labelled X, $\mathrm{XX}$, and Xc experience abrupt jumps in their energy position which moreover are strongly correlated, while other lines (as the one on the right-hand part of Fig. 4) do not jump or do it in a different way. As already reported [10, 11], this behavior can be interpreted as the fluctuations of the local electric field due to the capture or escape of charges trapped on defects in the neighborhood of a particular QD, which produces small Stark shift of the excitonic transitions. The power dependence we observed agrees well with this interpretation, since the stronger the optical excitation power, the greater the number of photocreated free carriers. The correlation in the shifts between different lines allows us to identify these lines 

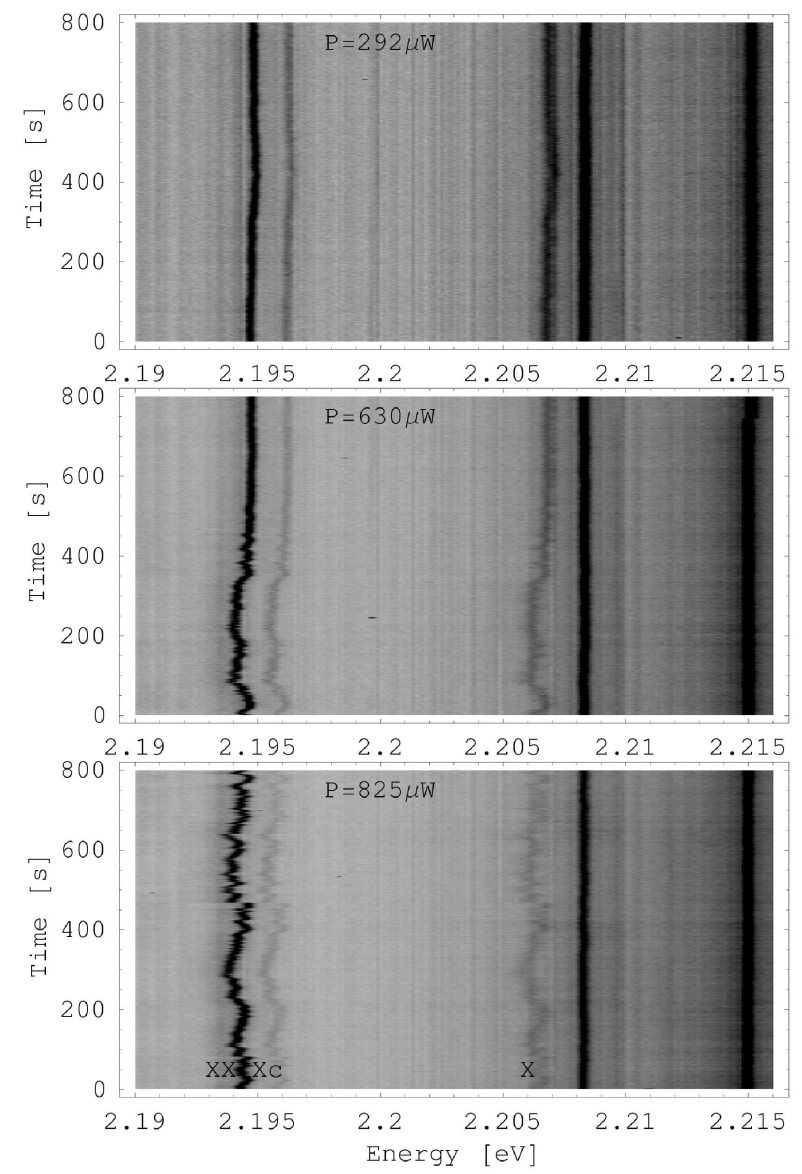

Fig. 4. Temporal evolution of the PL spectra of ZnTe/CdTe single QDs, at different excitation power $P$ measured in front of cryostat. Symbols: XX, Xc, and X denote biexciton, charged exciton, and exciton, respectively.

as different excitation states (exciton X, biexciton XX, and charged exciton Xc) of a unique QD. This interpretation is further supported by the energy splitting expected for these lines. Although this method does not permit to control properly the electric field experienced by a QD (neither in amplitude nor in direction), it offers an original way to investigate through a statistical analysis the relation between the Stark shift and the AES of a single QD, both for the exciton and biexciton lines. This will be the next step of the current study.

\section{Conclusions}

In this work we present experimental results on single QDs made of III-V and II-VI materials. It is shown that an electric field applied in the plane of InAs quantum dots can reduce significantly the anisotropic electron-hole exchange 
interaction of the excitonic ground states, which should be of great interest in order to produce entangled photon pairs with QDs. The (non-controlled) electric field fluctuations that are observed in the case of II-VI QDs under strong photoexcitation, could also be used to investigate these properties.

\section{Acknowledgment}

This work was supported by the State Committee for Scientific Research (Poland) (grants 2P03 B01525 and PBZ-KBN-044/P03/2001), by French-Polish collaboration program Polonium, by the Région Île de France and by Conseil Général de l'Essonne.

\section{References}

[1] R. Ferreira, A. Jankovic, private communication.

[2] S.V. Gupalov, E.L. Ivchenko, A.V. Kavokin, Sov. Phys. JETP 86, 388 (1998).

[3] M.Z. Maialle, E.A. de Andrada e Silva, L.J. Sham, Phys. Rev. B 47, 15776 (1993).

[4] B. Aléna, F. Bickel, K. Karrai, R.J. Warburton, P.M. Petroff, Appl. Phys. Lett. 83, 2235 (2004).

[5] P.W. Fry, I.E. Itskevich, D.J. Mowbray, M.S. Skolnick, J.J. Finley, J.A. Barker, E.P. O'Reilly, L.R. Wilson, I.A. Larkin, P.A. Maksym, M. Hopkinson, M. Al-Khafaji, J.P.R. David, A.G. Cullis, G. Hill, J.C. Clark, Phys. Rev. Lett. 84, $733(2000)$.

[6] G. Karczewski, S. Maćkowski, M. Kutrowski, T. Wojtowicz, J. Kossut, Appl. Phys. Lett. 74, 3011 (1999).

[7] K. Kowalik, A. Kudelski, A. Golnik, J.A. Gaj, G. Karczewski, J. Kossut, Acta Phys. Pol. A 103, 539 (2003).

[8] J. Jasny, J. Sepiol, T. Irngartinger, M. Traber, A. Renn, U.P. Wild, Rev. Sci. Instrum. 67, 1425 (1996).

[9] A. Kudelski, A. Golnik, J.A. Gaj, S. Maćkowski, G. Karczewski, J. Kossut, in: Proc. 25th Int. Conf. Phys. Semicond., Osaka 2000, Eds. N. Miura, T. Ando, Springer, Berlin 2001, p. 1249.

[10] V. Türk, S. Rodt, O. Stier, R. Heitz, R. Engelhardt, U.W. Pohl, D. Bimberg, R. Steingrüber, Phys. Rev. B 61, 9944 (2000).

[11] H.D. Robinson, B.B. Goldberg, Phys. Rev. B 61, R5086 (2000). 